\title{
A plant's diet, surviving in a variable nutrient environment
}

\author{
Giles E. D. Oldroyd $1,2 *$ and Ottoline Leyser 1
}

\begin{abstract}
Affiliations:
1Sainsbury Laboratory, University of Cambridge, 47 Bateman Street, Cambridge CB2 1LR

2Crop Science Centre, University of Cambridge, 93 Lawrence Weaver Road, Cambridge CB3 OLE

*Correspondence to: gedo2@cam.ac.uk

Abstract: As primary producers, plants rely on a large above ground surface area to collect carbon dioxide and sunlight and a large underground surface area to collect the water and mineral nutrients needed to support their growth and development. Accessibility of the essential nutrients nitrogen and phosphorus in the soil is impacted by many factors that create a variable spatiotemporal landscape of their availability both at the local and global scale. Plants optimize uptake of the nitrogen and phosphorus available through modifications to their growth and development and through the engagement with microorganisms that facilitate their capture. The sensing of these nutrients, as well as the perception of overall nutrient status shape the plant's response to its nutrient environment, coordinating its development with microbial engagement to optimize nitrogen and phosphorus capture and regulate overall plant growth.
\end{abstract}

One Sentence Summary: Plants have adapted to survive in a variable landscape of nutrient availability through changes in their development and adjustments in microbial engagement, controlled by common local and systemic signaling processes.

\section{Introduction}

While plants are dependent on the capture of a number of elemental nutrients from the soil, the principle nutrients that limit plant productivity are nitrogen $(\mathrm{N})$ and phosphorus $(\mathrm{P})$. Acquisition of these nutrients is essential for crop performance, but levels of these nutrients in most agricultural soils limit productivity. Thus, these nutrients are typically applied at high concentrations, in the form of inorganic fertilizers, to support global food production. However, the global supply of $\mathrm{P}$, which is sourced from rock mining, is finite and the manufacture of reactive $\mathrm{N}$ for fertilizer is currently dependent on fossil fuels to drive the energy-intensive HaberBosch process (1). Where farmers can afford fertilizers, their use is often profligate, and while this ensures crop productivity it also creates problems due to the environmental release of these nutrients $(2)$, which reduces biodiversity and contributes to climate change $(3,4)$. The opposing problem exists for small-holder farmers in developing nations, who generally lack the financial resources to utilize inorganic fertilizers, with their crop yields suffering as a consequence (5). A more sustainable and equitable agriculture will be less dependent on inorganic fertilizers than the current state of play.

Because of the central importance of $\mathrm{N}$ - and P-availability in both natural and agricultural ecosystems, we focus this review on plant perception, acquisition and response to these two nutrients. Where these nutrients are ample, the ratio of root:shoot biomass allocation can be low with minimal root systems capturing sufficient nutrients. Typically, vegetative growth is extended, allowing resource accumulation and investment in seed production. In environments where these nutrients are limiting overall growth is reduced, but root systems are expanded and 
colonization by microorganisms is encouraged, to facilitate nutrient capture. We now have a detailed knowledge of the processes that control the plant's response to $\mathrm{N}$ and $\mathrm{P}$ and in this review we attempt to summarize this understanding. Virtually all of the work we describe is derived from model plant systems and while there are ample opportunities to use this knowledge to improve sustainable productivity in agriculture, this requires extensive research in crops.

\section{Root developmental adaptations to $\mathbf{N}$-availability}

Plants are reliant on reactive forms of $\mathrm{N}$, of which nitrate $\left(\mathrm{NO}_{3}-\right)$ is the most prevalent in soils, although ammonium and amino acids can also be present $(6,7)$. N-limitation results in biomass allocation to the root at the expense of the shoot (Fig. 1). Where $\mathrm{N}$ supply is uniformly low, lateral root growth is promoted and when $\mathrm{N}$ is uniformly high, lateral root growth is suppressed (Fig. 1) (8). However, when $\mathrm{N}$ supply is uneven, lateral roots proliferate into local patches of high $\mathrm{N}(9)$ and this $\mathrm{N}$-foraging response has been well studied for $\mathrm{NO}_{3}$ - (10). These effects are particularly clear in split root experiments (11), in which the root system is divided at an early stage in development with different nutrient treatments supplied to the two halves of the root system feeding one plant (12). Lateral root growth is promoted when both compartments have low $\mathrm{N}$, but is suppressed in the low $\mathrm{N}$-compartment and promoted in the high $\mathrm{N}$-compartment in differentially treated plants (Fig. 1). The shoot is essential for mediating these root responses in split root systems, since the root response is lost following decapitation (12). These results contribute to a growing body of evidence suggesting that the root response to $\mathrm{N}$ involves four signaling processes: (1) local signaling in the root associated with perception of local N; (2) rootshoot-root signaling indicating the presence of roots experiencing low [N]; (3) root-shoot-root signaling indicating the presence of roots experiencing locally high [N]; and (4) a systemic inhibitory signal that suppresses root $\mathrm{N}$-foraging activities when shoot $[\mathrm{N}]$ is sufficient.

\section{Mechanisms of $\mathbf{N}$ sensing and response}

Signal 1: Local perception and uptake of $\mathrm{NO}_{3}$ -

Because $\mathrm{NO}_{3}$ - is an important $\mathrm{N}$-source, plants have developed processes for $\mathrm{NO}_{3}$ - uptake, as well as the ability to sense $\mathrm{NO}_{3}$ - directly at the plant surface (13). One protein, Nitrate Transporter (NRT)1.1, a plasma membrane transceptor, contributes to both high and low affinity NO3- uptake and acts as a receptor for the perception of NO3- (14-16). NRT1.1 controls responses to different $\mathrm{NO}_{3}$ - concentrations (17). A switch in NRT1.1 mode of action, controlled by phosphorylation (18), involves a suite of calcium-regulated kinases $(14,19)$, that dictates the dimerization state of the protein $(20,21)$, which switches this transceptor from a low to a high affinity $\mathrm{NO}_{3}$ - transporter (18) and affects the modality of signaling $(14,17)$.

At least three additional classes of $\mathrm{NO}_{3}$ - transporters contribute to low- and high-affinity uptake $(22,23)$. NRT1.1 controls the expression of these additional transporters, especially NRT2.1 (24), through a NO3--induced calcium influx into the cytosol and nucleus (Fig. 2) (25, 26). The calcium transient has two primary modes of action: further regulation of NRT1.1 (14, 27, 28) and induction of calcium-sensor protein kinases (Fig. 2) that phosphorylate the transcription factor NIN-like protein (NLP)7 (26). NLP7 is excluded from the nucleus, but NO3-induced phosphorylation drives NLP7 into the nucleus $(26,29)$, where it upregulates the MADS box transcription factor Arabidopsis Nitrate Response (ANR)1, that promotes lateral root proliferation into $\mathrm{NO}_{3}$-rich patches $(8)$ and nitrate transporters to drive further uptake of nitrate (29-31). 
Signal 2: Long distance signaling to indicate local $N$-depletion

$\mathrm{C}$-Terminally Encoded Peptides (CEPs) have emerged as regulators of systemic N-signaling (32). These peptides are produced in roots experiencing $\mathrm{N}$-limitation and activate $\mathrm{NO}_{3}-$ transporters, such as NRT2.1, in roots where NO3- is plentiful (Fig. 1) (33). CEP peptides travel to the shoot through the xylem, where recognition by CEP receptors (33) leads to production of a second class of peptides, CEPDs (34). CEPDs are produced in leaves but function in roots where they upregulate NRT2.1 expression in locations where $\mathrm{NO}_{3}$ - is ample (34). However, there is no preferential transport of CEPDs to roots expressing NRT2.1 (34), suggesting that additional signals must integrate with this systemic signal, to drive the local response. We suggest that the CEPD signal likely integrates with the local $\mathrm{NO}_{3}$ - response (signal 1; Fig. 2) to coordinate root growth into nitrate-rich compartments. The transcription factor TCP-domain family protein 20 (TCP20) has a function in the systemic N-response (35), that interacts with NLP6 and NLP7 (36) and is therefore a candidate to integrate the systemic and local N-responses (Fig. 2).

\section{Signal 3: Activating $N$-foraging in $N$-rich patches}

Cytokinin biosynthesis is promoted in roots perceiving high $\mathrm{NO}_{3}$ - (Fig. 1) (37) and translocated to the shoot, where it coordinates growth (38). Deficiency in cytokinin biosynthesis blocks the $\mathrm{N}$-foraging response but can be counteracted by addition of cytokinin even in roots under low-N (12). This suggests that cytokinin acts as a root-to-shoot signal from patches of high $\mathrm{N}$ availability and coordinates with the CEP-signal, since the effect of cytokinin only occurs when there are roots in low $\mathrm{NO}_{3}-(12,39)$. CEPD production may be directly or indirectly dependent on cytokinin (Fig. 1), allowing for integration of signals indicating locally low N-supply with signals indicating locally high $\mathrm{N}$-supply.

\section{Signal 4: Coordinating root growth with shoot $N$-status}

Plants also assess their overall $\mathrm{N}$-status (40) and use the information to modulate growth and metabolism as well as to balance carbon (C)- and $\mathrm{N}$-acquisition. Amino acids or $\mathrm{NO}_{3}$ - may act as proxies for $\mathrm{N}$ status (41-43). Although amino acids and $\mathrm{NO}_{3}$ - contribute to $\mathrm{N}$-status sensing (44), there is an intrinsic problem with using these alone to signal global N-status. Doing so is equivalent to assessing one's financial situation based only on the current account balance: projected income, expenditure and savings all add to the demand for $\mathrm{N}$ and must be considered when calculating responses to nutrient environments. The $\mathrm{N}$-sufficiency signal (possibly relative to C-supply) must integrate with the CEP and cytokinin signals to block $\mathrm{N}$-foraging when shoot $\mathrm{N}$ is sufficient $(8,44)$.

\section{Root developmental adaptations to $\mathbf{P}$-availability}

As for $\mathrm{N}$ supply, plants also adjust their root system architecture in response to $\mathrm{P}(45,46)$, primarily available in soil as phosphate ( $\left.\mathrm{PO}_{4}-\right)$. As with $\mathrm{NO}_{3}$-, there are different responses to $\mathrm{PO} 4-$ whether spatially uniform or supplied heterogeneously. While the roots of $\mathrm{PO} 4$ - deficient plants proliferate into patches of high $\mathrm{P}$ (47), in a similar way to $\mathrm{N}$ (48), their responses to uniformly low $\mathrm{P}$ are significantly different. Under uniformly low $\mathrm{PO}_{4}$, primary root growth is repressed, lateral root growth promoted and root hairs elongated, but total root system length remains remarkably constant (47). These differences are consistent with $\mathrm{PO}_{4}$ - having limited mobility in soil, with greatest availability in topsoil, whereas $\mathrm{NO}_{3}$ - is freely mobile (49). Dense foraging in topsoil improves $\mathrm{PO}_{4}$ - capture, while low density exploration of a large soil volume improves $\mathrm{NO}_{3}$ - capture. Such dynamic root responses are important for P-capture: suppressing 
lateral root production does not impede competitiveness under $\mathrm{N}$-limitation, but does under $\mathrm{P}$ limitation (50). PO4- has systemic effects on root development (47), but gene expression in split roots suggests root growth is primarily regulated by local $\mathrm{PO}_{4-}$, whereas systemic $\mathrm{P}$-signaling regulates genes associated with $\mathrm{PO}_{4}$ - uptake and assimilation (51).

\section{Mechanisms of $\mathbf{P}$-sensing and response}

Responses to local sources of $\mathrm{PO}_{4}$ -

P-regulation of root system architecture is driven by the local perception of $\mathrm{PO}_{4}$ - at the root tip (52) and involves changes in multiple plant hormones (Fig. 3). Primary among these are complex patterns of auxin redistribution: increased auxin levels in primary root and young lateral root tips, but decreased auxin levels in older lateral root tips $(53,54)$. These auxin dynamics function alongside other P-starvation signals to coordinate root development: strigolactones (55-57), the peptide hormones Root Growth Factor (RGF)1 and RGF2 (58); DELLA-domain containing proteins that accumulate under the declining concentrations of gibberellins (59) and ethylene, which controls root hair elongation (60-63). These hormonal changes must together coordinate the root developmental responses to P-availability, but the mechanisms of integration need to be established.

\section{Systemic signaling under P-limitation}

Systemic signaling between the root and the shoot is implicated in P-regulation of shoot growth and the regulation of $\mathrm{PO}_{4}$ - transporters (45). $\mathrm{PO}_{4}$ - itself is implicated as a systemic signaling molecule (64), but as outlined for $\mathrm{N}$, additional signals are required for the plant to ascertain its full P-status and needs. Strigolactones act as systemic P-availability signals (56), coordinating shoot growth (65), while cytokinins are downregulated under P-starvation (66), to remove a transcriptional repression $(67,68)$. The systemic P-starvation transcriptional response is induced by the transcription factor Phosphate starvation response (PHR) 1 and its homologs $(69,70)$, that are regulated by $\mathrm{PO}_{4-}$, through the availability of phytate (71-73) (Fig. 3). PHRI contributes to the systemic regulation of $\mathrm{PO}_{4}$ - transporters in roots (74-76) through shoot derived microRNAs (77): miR399 $(78-80)$ and miR827 $(81,82)$, that can be regulated by microRNA mimics $(83)$.

\section{Microbes to the rescue under nutrient deprivation}

Plant engagement with microorganisms can improve nutrient acquisition: mutualistic arbuscular mycorrhizal associations increase the surface area for $\mathrm{PO}_{4}$ - and $\mathrm{NO}_{3}$ - capture, while symbiotic nitrogen-fixing bacteria convert $\mathrm{N}_{2}$ into $\mathrm{NH}_{4+}$. The arbuscular mycorrhizal association dates to the earliest land plants (84). In these early land plants the fungal association likely provided the primary interface for nutrient capture, facilitating the transition from an aquatic to a terrestrial lifestyle (84). Because of the early origin for this symbiosis, the arbuscular mycorrhizal association is widespread in the plant kingdom and intricately networked with plant nutrient capture physiology. The evolutionary processes that underpinned the emergence of arbuscular mycorrhizal associations also facilitated the evolution of other intracellular symbioses (85). However, independent losses of these symbioses, associated with loss of the underpinning genetic networks (86-89), suggests a cost for these symbioses, with negative selection in environments or plant lifestyles where microbial nutrient services are no longer advantageous. Supporting these microbes' energy needs (90), as well as the plants' need to suppress immunity to facilitate symbiont colonization (91) may drive selection against these associations when they 
are no longer of value. The molecular components that guide plant developmental responses to the nutrient environment also coordinate engagement with microorganisms.

Regulation of $\mathrm{N}$-fixing associations

Mutualistic bacterial associations facilitate $\mathrm{N}$-capture through the fixation of $\mathrm{N}_{2}$ by the bacterial enzyme nitrogenase. Many such $\mathrm{N}$-fixing bacterial associations have evolved across the plant kingdom, ranging from intracellular colonization of specialized root organs, known as nodules (92), to bacteria existing outside the root in polysaccharide matrices (93). The most effective associations, demonstrated by legumes, involve intimate engagement between intracellular Nfixing bacteria housed inside membrane-bound compartments within root nodule cells (92). Nodules allow a gaseous environment to be created that optimizes $\mathrm{N}$-capture and facilitates integration of plant and bacterial metabolism ensuring ammonia released to the plant (94).

Because of the high energetic costs of bacterial $\mathrm{N}$-fixation, this process generally only benefits the plant when it is starved for $\mathrm{N}$ but has ample $\mathrm{C}(95,96)$. Thus the association is regulated according to both $\mathrm{N}$ and $\mathrm{C}$-availability (97). Nodulation is controlled by the CEP peptides and their receptors (98-100), suggesting that as plants evolved $\mathrm{N}$-fixing capabilities they linked the decision to engage with these symbionts to existing low-N signals. In addition plants evolved a second systemic signaling process to regulate the total level of $\mathrm{N}$-fixation, so-called autoregulation of nodulation (101) (Fig. 4). This system restricts further engagement with Nfixing bacteria after a first round of colonization, but also regulates this process according to the availability of $\mathrm{NO}_{3}$ - (101). Here, CLE peptides are produced in the root following recognition of $\mathrm{N}$-fixing bacteria, then transported to the shoot, where receptor recognition leads to the suppression of miR2111 expression, that acts as a shoot-root signal to inhibit a negative regulator of nodulation $(101,102)$. Thus high levels of miR2111 create a symbiotically permissive state, while inhibition of miR2111 expression switches the plant to a symbiotically restrictive state (Fig. 4). This systemic signaling process is linked to local NO3-perception through NIN-like proteins that also activate the expression of CLE peptides in the root and can block aspects of nodulation signaling $(103,104)$. Thus regulation of $\mathrm{N}$-fixation has integrated both local and systemic processes that control plant responses to $\mathrm{N}$-availability.

\section{Regulation of arbuscular mycorrhizal associations}

Arbuscular mycorrhizal fungi are dependent on their host plant for C (90), making PO4--uptake through this association costly and hence regulated according to P-availability (105). Evidence is emerging that the mechanisms controlling systemic autoregulation of nodulation also regulate mycorrhization $(101,106)$, implicating miR2111. Indeed, miR2111 is induced under P-starvation (82). The role of miR2111 in regulating $\mathrm{N}$-fixation may be another manifestation of the recruitment of pre-existing plant-mycorrhizal signaling to nodulation (85). Additional Pstarvation induced components also regulate mycorrhizal colonization: DELLAs, which accumulate in the absence of gibberellins (the situation under low-P), are positive regulators of mycorrhization (107) and strigolactones act as plant signals to mycorrhizal fungi (108), promoting fungal processes necessary for the interaction. A signaling process related to that of strigolactones, but involving karrikin-like molecules, is essential for mycorrhizal colonisation (109), but there is no evidence yet that karrikin-like signals are associated with plant nutrient status. It appears that multiple apocarotenoid molecules, including strigolactones and perhaps karrikin-like, coordinate plant development and microbial associations (110) and there may be other unknown signaling molecules in this class of small metabolites. Once established the level 
of $\mathrm{PO}_{4}$ - supply by the fungus is monitored and if insufficient $\mathrm{PO}_{4}$ - is forthcoming, the plant blocks the fungal association (111). Similarly, N supply from $\mathrm{N}$-fixing associations is monitored (112). Such constant substantiation of the delivery of nutrients helps limit the emergence of microbial cheaters that might colonize the root to gain benefits without providing nutrient services.

\section{Coordinating the root microbiome with plant $P$ status}

Plants engage with a diversity of microorganisms in the rhizosphere. Some of these commensal associations can facilitate nutrient acquisition. The demonstration that a species of Colletotrichum can confer plant fitness (113) highlights the delicate balance between mutualist and pathogenic lifestyles, since many closely related species of Colletotrichum are plant pathogens. Facilitation of plant acquisition of $\mathrm{PO}_{4}$ - can be via direct delivery of $\mathrm{PO}_{4}$, as in the case of Colletotrichum tofeldiae (113), or may be due to the reprogramming of the plant's $\mathrm{PO}_{4}$ uptake machinery upon fungal colonisation, as proposed for Piriformospora indica (114). These commensal associations are also regulated by components that control the plants' broader response to its nutrient status: $C$. tofeldiae infection and rhizosphere bacterial communities are regulated by $\mathrm{P}$-status in a manner dependent on PHR1 $(113,115)$. P-starvation is associated with the suppression of immunity in Arabidopsis (115), implying that plants are willing to take risks under nutrient starvation in order to facilitate colonization by associative microorganisms to help in the capture of nutrients.

\section{Integration}

A focus on single nutrient treatments is a key limitation to many of the studies that have led to our current understanding of the plant response to its nutrient environment. N- and P-availability are not alone in directing the degree of plant performance, other factors such as light, water, other nutrients and prevalence of pests and pathogens, all dictate the way in which the plant should partition resources to optimize performance. Plants coordinate their developmental and microbial responses to $\mathrm{N}$ - and $\mathrm{P}$-availability with their capacity to fix $\mathrm{C}$. For example, light intensity can be relayed by the phytochrome B-responsive transcription factor Elongated Hypocotyl (HY)5, which acts as a mobile shoot-to-root signal that regulates $\mathrm{N}$ and $\mathrm{P}$ acquisition $(116,117)$. HY5 has functions in both root developmental adaptations and the regulation of symbiotic N-fixation $(116,118)$. Similarly, plant responses to water availability are modulated by the availability of $\mathrm{N}$ (119) and the water stress signaling system that functions through abscisic acid directly affects the local root response to NO3- (19). The plant is reliant and responsive to a wide array of nutrients (120) and these must be measured in combination to optimize plant performance. $\mathrm{N}$ - and P-responses are integrated (121) and affected by the availability of a range of additional nutrients (122), but the underlying mechanisms for such nutrient coordination are only just beginning to emerge (123-125).

\section{Conclusions}

Plants have adapted to survive spatiotemporally variable environments, including the variable landscape of nutrient accessibility. A combination of local nutrient sensing in the root with systemic signaling integrated in the shoot creates the response to the nutrient landscape, whether low or high in nutrient content, patchy or continuous. Under nutrient deprivation, plants turn to microorganisms to help facilitate nutrient capture through processes linked to developmental adaptations to nutrient availability. $\mathrm{N}$ and $\mathrm{P}$ are critical for global food production. Opportunities 
exist to adapt crop performance such that $\mathrm{N}$ and $\mathrm{P}$-use is improved even as environmental sustainability is enhanced. Although our understanding of how plants engage with their nutrients has advanced, few examples exist where such knowledge has impacted plant performance and this is almost certainly related to the fact that virtually all our understanding described in this review derives from studies in model, not crop, plants. Years of plant breeding under highnutrient environments have left us with some crop varieties that are poor at optimizing use of limited nutrients (126). Nonetheless, many processes exist in plants to ensure productivity under poor nutrient conditions, some of which are already accessible in the diversity of crop species (127). It is time we applied the deep understanding in model plants to impart improvements of crops to wean the global population from its dependency on inorganic fertilizers.

\section{References}

1. W. F. Zhang et al., New technologies reduce greenhouse gas emissions from nitrogenous fertilizer in China. Proc Natl Acad Sci U S A 110, 8375-8380 (2013).

2. C. J. Stevens, Nitrogen in the environment. Science 363, 578-580 (2019).

3. J. A. Foley et al., Soultions for a cultivated planet. Nature 478, 337-342 (2011).

4. J. Rockstrom et al., A safe operating space for humanity. Nature 461, 472-475 (2009).

5. N. D. Mueller et al., Closing yield gaps through nutrient and water management. Nature 490, 254-257 (2012).

6. L. Gent, B. G. Forde, How do plants sense their nitrogen status? J Exp Bot 68, 2531-2539 (2017).

7. S. Filleur, P. Walch-Liu, Y. Gan, B. G. Forde, Nitrate and glutamate sensing by plant roots. Biochem Soc T 33, 283-286 (2005).

8. H. Zhang, B. G. Forde, An Arabidopsis MADS box gene that controls nutrient-induced changes in root architecture. Science 279, 407-409 (1998).

9. M. C. Drew, Comparison of Effects of a Localized Supply of Phosphate, Nitrate, Ammonium and Potassium on Growth of Seminal Root System, and Shoot, in Barley. New Phytologist 75, 479-490 (1975).

10. F. Bellegarde, A. Gojon, A. Martin, Signals and players in the transcriptional regulation of root responses by local and systemic N signaling in Arabidopsis thaliana. J Exp Bot 68, 2553-2565 (2017).

11. L. E. Nelson, N. C. Brady, Some Greenhouse Studies of Cation Interactions in Ladino Clover Using Split Root Techniques. Soil Sci Soc Am Pro 17, 274-278 (1953).

12. S. Ruffel et al., Nitrogen economics of root foraging: Transitive closure of the nitratecytokinin relay and distinct systemic signaling for N supply vs. demand. Proceedings of the National Academy of Sciences of the United States of America 108, 18524-18529 (2011).

13. P. Walch-Liu, S. Filleur, Y. B. Gan, B. G. Forde, Signaling mechanisms integrating root and shoot responses to changes in the nitrogen supply. Photosynth Res 83, 239-250 (2005).

14. C. H. Ho, S. H. Lin, H. C. Hu, Y. F. Tsay, CHL1 Functions as a Nitrate Sensor in Plants. Cell 138, 1184-1194 (2009).

15. C. H. Ho, Y. F. Tsay, Nitrate, ammonium, and potassium sensing and signaling. Current Opinion in Plant Biology 13, 604-610 (2010).

16. Y. F. Tsay, J. I. Schroeder, K. A. Feldmann, N. M. Crawford, The Herbicide Sensitivity Gene Chl1 of Arabidopsis Encodes a Nitrate-Inducible Nitrate Transporter. Cell 72, 705713 (1993). 
17. E. Bouguyon et al., Multiple mechanisms of nitrate sensing by Arabidopsis nitrate transceptor NRT1.1. Nat Plants 1, (2015).

18. K. H. Liu, Y. F. Tsay, Switching between the two action modes of the dual-affinity nitrate transporter CHL1 by phosphorylation. Embo Journal 22, 1005-1013 (2003).

19. S. Leran et al., Nitrate sensing and uptake in Arabidopsis are enhanced by ABI2, a phosphatase inactivated by the stress hormone abscisic acid. Sci Signal 8, (2015).

20. J. L. Parker, S. Newstead, Molecular basis of nitrate uptake by the plant nitrate transporter NRT1.1. Nature 507, 68-72 (2014).

21. J. Sun et al., Crystal structure of the plant dual-affinity nitrate transporter NRT1.1. Nature 507, 73-77 (2014).

22. A. De Angeli et al., Review. CLC-mediated anion transport in plant cells. Philos Trans $R$ Soc Lond B Biol Sci 364, 195-201 (2009).

23. B. G. Forde, Nitrate transporters in plants: structure, function and regulation. Biochim Biophys Acta 1465, 219-235 (2000).

24. S. Munos et al., Transcript profiling in the chl1-5 mutant of Arabidopsis reveals a role of the nitrate transporter NRT1.1 in the regulation of another nitrate transporter, NRT2.1. Plant Cell 16, 2433-2447 (2004).

25. E. Riveras et al., The Calcium Ion Is a Second Messenger in the Nitrate Signaling Pathway of Arabidopsis. Plant Physiol 169, 1397-1404 (2015).

26. K. H. Liu et al., Discovery of nitrate-CPK-NLP signalling in central nutrient-growth networks. Nature 545, 311-316 (2017).

27. H. C. Hu, Y. Y. Wang, Y. F. Tsay, AtCIPK8, a CBL-interacting protein kinase, regulates the low-affinity phase of the primary nitrate response. Plant Journal 57, 264-278 (2009).

28. S. Leran et al., AtNPF5.5, a nitrate transporter affecting nitrogen accumulation in Arabidopsis embryo. Sci Rep-Uk 5, (2015).

29. C. Marchive et al., Nuclear retention of the transcription factor NLP7 orchestrates the early response to nitrate in plants. Nat Commun 4, 1713 (2013).

30. L. Castaings et al., The nodule inception-like protein 7 modulates nitrate sensing and metabolism in Arabidopsis. Plant J 57, 426-435 (2009).

31. M. Konishi, S. Yanagisawa, Arabidopsis NIN-like transcription factors have a central role in nitrate signalling. Nat Commun 4, 1617 (2013).

32. M. Taleski, N. Imin, M. A. Djordjevic, CEP peptide hormones: key players in orchestrating nitrogen-demand signalling, root nodulation, and lateral root development. J Exp Bot 69, 1829-1836 (2018).

33. R. Tabata et al., Perception of root-derived peptides by shoot LRR-RKs mediates systemic N-demand signaling. Science 346, 343-346 (2014).

34. Y. Ohkubo, M. Tanaka, R. Tabata, M. Ogawa-Ohnishi, Y. Matsubayashi, Shoot-to-root mobile polypeptides involved in systemic regulation of nitrogen acquisition. Nat Plants 3, 17029 (2017).

35. P. Guan et al., Nitrate foraging by Arabidopsis roots is mediated by the transcription factor TCP20 through the systemic signaling pathway. Proc Natl Acad Sci U S A 111, 15267-15272 (2014).

36. P. Guan et al., Interacting TCP and NLP transcription factors control plant responses to nitrate availability. Proc Natl Acad Sci U S A 114, 2419-2424 (2017).

37. H. Sakakibara, K. Takei, N. Hirose, Interactions between nitrogen and cytokinin in the regulation of metabolism and development. Trends Plant Sci 11, 440-448 (2006). 
38. B. Landrein et al., Nitrate modulates stem cell dynamics in Arabidopsis shoot meristems through cytokinins. Proc Natl Acad Sci U S A 115, 1382-1387 (2018).

39. A. Poitout et al., Responses to Systemic Nitrogen Signaling in Arabidopsis Roots Involve trans-Zeatin in Shoots. Plant Cell 30, 1243-1257 (2018).

40. X. Gansel, S. Munos, P. Tillard, A. Gojon, Differential regulation of the NO3- and NH4+ transporter genes AtNrt2.1 and AtAmt1.1 in Arabidopsis: relation with long-distance and local controls by N status of the plant. Plant J 26, 143-155 (2001).

41. J. Imsande, B. Touraine, N Demand and the Regulation of Nitrate Uptake. Plant Physiol 105, 3-7 (1994).

42. L. Lejay et al., Molecular and functional regulation of two NO3- uptake systems by Nand C-status of Arabidopsis plants. Plant J 18, 509-519 (1999).

43. M. L. Gifford, A. Dean, R. A. Gutierrez, G. M. Coruzzi, K. D. Birnbaum, Cell-specific nitrogen responses mediate developmental plasticity. Proc Natl Acad Sci U S A 105, 803808 (2008).

44. H. Zhang, A. Jennings, P. W. Barlow, B. G. Forde, Dual pathways for regulation of root branching by nitrate. Proc Natl Acad Sci U S A 96, 6529-6534 (1999).

45. P. S. Chien, C. P. Chiang, S. J. Leong, T. J. Chiou, Sensing and Signaling of Phosphate Starvation - from Local to Long Distance. Plant Cell Physiol, (2018).

46. H. Crombez, H. Motte, T. Beeckman, Tackling Plant Phosphate Starvation by the Roots. Dev Cell 48, 599-615 (2019).

47. L. C. Williamson, S. P. Ribrioux, A. H. Fitter, H. M. Leyser, Phosphate availability regulates root system architecture in Arabidopsis. Plant Physiol 126, 875-882 (2001).

48. B. I. Linkohr, L. C. Williamson, A. H. Fitter, H. M. Leyser, Nitrate and phosphate availability and distribution have different effects on root system architecture of Arabidopsis. Plant J 29, 751-760 (2002).

49. P. B. Tinker, P. H. Nye, Solute movement in the rhizosphere. Topics in sustainable agronomy (Oxford University Press, New York, 2000), pp. xix, 444 p.

50. A. Fitter, L. Williamson, B. Linkohr, O. Leyser, Root system architecture determines fitness in an Arabidopsis mutant in competition for immobile phosphate ions but not for nitrate ions. Proceedings. Biological sciences / The Royal Society 269, 2017-2022 (2002).

51. M. C. Thibaud et al., Dissection of local and systemic transcriptional responses to phosphate starvation in Arabidopsis. Plant J 64, 775-789 (2010).

52. S. Svistoonoff et al., Root tip contact with low-phosphate media reprograms plant root architecture. Nat Genet 39, 792-796 (2007).

53. Y. Al-Ghazi et al., Temporal responses of Arabidopsis root architecture to phosphate starvation: evidence for the involvement of auxin signalling. Plant Cell and Environment 26, 1053-1066 (2003).

54. P. Nacry et al., A role for auxin redistribution in the responses of the root system architecture to phosphate starvation in Arabidopsis. Plant Physiology 138, 2061-2074 (2005).

55. M. Lopez-Obando, Y. Ligerot, S. Bonhomme, F. D. Boyer, C. Rameau, Strigolactone biosynthesis and signaling in plant development. Development 142, 3615-3619 (2015).

56. M. Umehara et al., Inhibition of shoot branching by new terpenoid plant hormones. Nature 455, 195-U129 (2008).

57. Y. Kapulnik et al., Strigolactones affect lateral root formation and root-hair elongation in Arabidopsis. Planta 233, 209-216 (2011). 
58. H. M. Cederholm, P. N. Benfey, Distinct sensitivities to phosphate deprivation suggest that RGF peptides play disparate roles in Arabidopsis thaliana root development. New Phytologist 207, 683-691 (2015).

59. C. F. Jiang, X. H. Gao, L. Liao, N. P. Harberd, X. D. Fu, Phosphate starvation root architecture and anthocyanin accumulation responses are modulated by the gibberellinDELLA signaling pathway in Arabidopsis(1[OA]). Plant Physiology 145, 1460-1470 (2007).

60. K. Borch, T. J. Bouma, J. P. Lynch, K. M. Brown, Ethylene: a regulator of root architectural responses to soil phosphorus availability. Plant Cell and Environment 22, 425-431 (1999).

61. K. M. Brown, K. Borch, J. P. Lynch, Ethylene mediation of low-phosphorus responses in roots. Phosphorus in Plant Biology: Regulatory Roles in Molecular, Cellular, Organismic, and Ecosystem Processes 19, 344-346 (1999).

62. K. Yi, B. Menand, E. Bell, L. Dolan, A basic helix-loop-helix transcription factor controls cell growth and size in root hairs. Nature Genetics 42, 264-U108 (2010).

63. L. Song et al., The Molecular Mechanism of Ethylene-Mediated Root Hair Development Induced by Phosphate Starvation. Plos Genet 12, (2016).

64. D. K. Varadarajan, A. S. Karthikeyan, P. D. Matilda, K. G. Raghothama, Phosphite, an analog of phosphate, suppresses the coordinated expression of genes under phosphate starvation. Plant Physiology 129, 1232-1240 (2002).

65. W. Kohlen et al., Strigolactones Are Transported through the Xylem and Play a Key Role in Shoot Architectural Response to Phosphate Deficiency in Nonarbuscular Mycorrhizal Host Arabidopsis. Plant Physiology 155, 974-987 (2011).

66. J. M. Franco-Zorrilla, A. C. Martin, A. Leyva, J. P. Par-Ares, Interaction between phosphate-starvation, sugar, and cytokinin signaling in arabidopsis and the roles of cytokinin receptors CRE1/AHK4 and AHK3. Plant Physiology 138, 847-857 (2005).

67. A. C. Martin et al., Influence of cytokinins on the expression of phosphate starvation responsive genes in Arabidopsis. Plant Journal 24, 559-567 (2000).

68. X. M. Wang et al., Cytokinin represses phosphate-starvation response through increasing of intracellular phosphate level. Plant Cell and Environment 29, 1924-1935 (2006).

69. R. Bustos et al., A Central Regulatory System Largely Controls Transcriptional Activation and Repression Responses to Phosphate Starvation in Arabidopsis. Plos Genet 6, (2010).

70. V. Rubio et al., A conserved MYB transcription factor involved in phosphate starvation signaling both in vascular plants and in unicellular algae. Gene Dev 15, 2122-2133 (2001).

71. M. I. Puga et al., SPX1 is a phosphate-dependent inhibitor of PHOSPHATE STARVATION RESPONSE 1 in Arabidopsis. Proceedings of the National Academy of Sciences of the United States of America 111, 14947-14952 (2014).

72. Z. Y. Wang et al., Rice SPX1 and SPX2 inhibit phosphate starvation responses through interacting with PHR2 in a phosphate-dependent manner. Proceedings of the National Academy of Sciences of the United States of America 111, 14953-14958 (2014).

73. R. Wild et al., Control of eukaryotic phosphate homeostasis by inositol polyphosphate sensor domains. Science 352, 986-990 (2016).

74. T. Y. Liu et al., PHO2-Dependent Degradation of PHO1 Modulates Phosphate Homeostasis in Arabidopsis. Plant Cell 24, 2168-2183 (2012). 
75. T. K. Huang et al., Identification of Downstream Components of Ubiquitin-Conjugating Enzyme PHOSPHATE2 by Quantitative Membrane Proteomics in Arabidopsis Roots. Plant Cell 25, 4044-4060 (2013).

76. A. K. Huen, C. Rodriguez-Medina, A. Y. Y. Ho, C. A. Atkins, P. M. C. Smith, Longdistance movement of phosphate starvation-responsive microRNAs in Arabidopsis. Plant Biology 19, 643-649 (2017).

77. M. I. Puga et al., Novel signals in the regulation of Pi starvation responses in plants: facts and promises. Curr Opin Plant Biol 39, 40-49 (2017).

78. T. J. Chiou et al., Regulation of phosphate homeostasis by microRNA in Arabidopsis. Plant Cell 18, 412-421 (2006).

79. S. I. Lin et al., Regulatory network of microRNA399 and PHO2 by systemic signaling. Plant Physiology 147, 732-746 (2008).

80. K. Aung et al., pho2, a phosphate overaccumulator, is caused by a nonsense mutation in a MicroRNA399 target gene. Plant Physiology 141, 1000-1011 (2006).

81. W. Y. Lin, T. K. Huang, T. J. Chiou, NITROGEN LIMITATION ADAPTATION, a Target of MicroRNA827, Mediates Degradation of Plasma Membrane-Localized Phosphate Transporters to Maintain Phosphate Homeostasis in Arabidopsis. Plant Cell 25, 4061-4074 (2013).

82. L. C. Hsieh et al., Uncovering Small RNA-Mediated Responses to Phosphate Deficiency in Arabidopsis by Deep Sequencing. Plant Physiology 151, 2120-2132 (2009).

83. J. M. Franco-Zorrilla et al., Target mimicry provides a new mechanism for regulation of microRNA activity. Nature Genetics 39, 1033-1037 (2007).

84. M. Parniske, Arbuscular mycorrhiza: the mother of plant root endosymbioses. Nature Reviews Microbiology 6, 763-775 (2008).

85. K. Markmann, M. Parniske, Evolution of root endosymbiosis with bacteria: how novel are nodules? Trends in Plant Science 14, 77-86 (2009).

86. A. Bravo, T. York, N. Pumplin, L. A. Mueller, M. J. Harrison, Genes conserved for arbuscular mycorrhizal symbiosis identified through phylogenomics. Nat Plants 2, 15208 (2016).

87. P. M. Delaux et al., Comparative phylogenomics uncovers the impact of symbiotic associations on host genome evolution. Plos Genet 10, e1004487 (2014).

88. M. Griesmann et al., Phylogenomics reveals multiple losses of nitrogen-fixing root nodule symbiosis. Science 361, (2018).

89. R. van Velzen et al., Comparative genomics of the nonlegume Parasponia reveals insights into evolution of nitrogen-fixing rhizobium symbioses. Proc Natl Acad Sci U S A 115, E4700-E4709 (2018).

90. L. H. Luginbuehl et al., Fatty acids in arbuscular mycorrhizal fungi are synthesized by the host plant. Science 356, 1175-1178 (2017).

91. Y. Liang et al., Nonlegumes respond to rhizobial Nod factors by suppressing the innate immune response. Science 341, 1384-1387 (2013).

92. G. E. D. Oldroyd, J. D. Murray, P. S. Poole, J. A. Downie, The Rules of Engagement in the Legume-Rhizobial Symbiosis. Annual Review Genetics, Vol 45 45, 119-144 (2011).

93. A. Van Deynze et al., Nitrogen fixation in a landrace of maize is supported by a mucilage-associated diazotrophic microbiota. PLoS Biol 16, e2006352 (2018).

94. E. M. Lodwig et al., Amino-acid cycling drives nitrogen fixation in the legumeRhizobium symbiosis. Nature 422, 722-726 (2003). 
95. D. J. Ballhorn, M. Schadler, J. D. Elias, J. A. Millar, S. Kautz, Friend or Foe-Light Availability Determines the Relationship between Mycorrhizal Fungi, Rhizobia and Lima Bean (Phaseolus lunatus L.). Plos One 11, (2016).

96. A. Rogers et al., Increased $\mathrm{C}$ availability at elevated carbon dioxide concentration improves N assimilation in a legume. Plant Cell and Environment 29, 1651-1658 (2006).

97. B. N. Taylor, D. N. L. Menge, Light regulates tropical symbiotic nitrogen fixation more strongly than soil nitrogen. Nat Plants 4, 655-661 (2018).

98. N. Imin, N. A. Mohd-Radzman, H. A. Ogilvie, M. A. Djordjevic, The peptide-encoding CEP1 gene modulates lateral root and nodule numbers in Medicago truncatula. J Exp Bot 64, 5395-5409 (2013).

99. N. A. Mohd-Radzman et al., Different Pathways Act Downstream of the CEP Peptide Receptor CRA2 to Regulate Lateral Root and Nodule Development. Plant Physiology 171, 2536-2548 (2016).

100. E. Huault et al., Local and Systemic Regulation of Plant Root System Architecture and Symbiotic Nodulation by a Receptor-Like Kinase. Plos Genet 10, (2014).

101. C. L. Wang, J. B. Reid, E. Foo, The Art of Self-Control - Autoregulation of PlantMicrobe Symbioses. Front Plant Sci 9, (2018).

102. D. Tsikou et al., Systemic control of legume susceptibility to rhizobial infection by a mobile microRNA. Science 362, 233-235 (2018).

103. J. S. Lin et al., NIN interacts with NLPs to mediate nitrate inhibition of nodulation in Medicago truncatula (vol 9, pg 942, 2018). Nat Plants 4, 1125-1125 (2018).

104. H. Nishida et al., A NIN-LIKE PROTEIN mediates nitrate-induced control of root nodule symbiosis in Lotus japonicus. Nat Commun 9, (2018).

105. L. M. Muller, M. J. Harrison, Phytohormones, miRNAs, and peptide signals integrate plant phosphorus status with arbuscular mycorrhizal symbiosis. Current Opinion in Plant Biology 50, 132-139 (2019).

106. L. M. Muller et al., A CLE-SUNN module regulates strigolactone content and fungal colonization in arbuscular mycorrhiza. Nat Plants 5, 933-939 (2019).

107. D. S. Floss, J. G. Levy, V. Levesque-Tremblay, N. Pumplin, M. J. Harrison, DELLA proteins regulate arbuscule formation in arbuscular mycorrhizal symbiosis. Proc Natl Acad Sci U S A 110, E5025-5034 (2013).

108. K. Akiyama, K. Matsuzaki, H. Hayashi, Plant sesquiterpenes induce hyphal branching in arbuscular mycorrhizal fungi. Nature 435, 824-827 (2005).

109. C. Gutjahr et al., Rice perception of symbiotic arbuscular mycorrhizal fungi requires the karrikin receptor complex. Science 350, 1521-1524 (2015).

110. J. Y. Wang et al., The apocarotenoid metabolite zaxinone regulates growth and strigolactone biosynthesis in rice. Nat Commun 10, 810 (2019).

111. H. Javot, R. V. Penmetsa, N. Terzaghi, D. Cook, M. J. Harrison, A Medicago truncatula phosphate transporter indispensable for the arbuscular mycorrhizal symbiosis. Proc Natl Acad Sci U S A 104, 1720-1725 (2007).

112. E. T. Kiers, R. A. Rousseau, S. A. West, R. F. Denison, Host sanctions and the legumerhizobium mutualism. Nature 425, 78-81 (2003).

113. K. Hiruma et al., Root Endophyte Colletotrichum tofieldiae Confers Plant Fitness Benefits that Are Phosphate Status Dependent. Cell 165, 464-474 (2016). 
114. M. Bakshi et al., Piriformospora indica Reprograms Gene Expression in Arabidopsis Phosphate Metabolism Mutants But Does Not Compensate for Phosphate Limitation. Frontiers in Microbiology 8, (2017).

115. G. Castrillo et al., Root microbiota drive direct integration of phosphate stress and immunity. Nature 543, 513-+ (2017).

116. X. B. Chen et al., Shoot-to-Root Mobile Transcription Factor HY5 Coordinates Plant Carbon and Nitrogen Acquisition. Current Biology 26, 640-646 (2016).

117. Y. Sakuraba et al., A phytochrome-B-mediated regulatory mechanism of phosphorus acquisition. Nat Plants 4, 1089-1101 (2018).

118. R. Nishimura, M. Ohmori, H. Fujita, M. Kawaguchi, A Lotus basic leucine zipper protein with a RING-finger motif negatively regulates the developmental program of nodulation. Proc Natl Acad Sci U S A 99, 15206-15210 (2002).

119. J. Swift, M. Adame, D. Tranchina, A. Henry, G. M. Coruzzi, Water impacts nutrient dose responses genome-wide to affect crop production. Nat Commun 10, (2019).

120. B. D. Gruber, R. F. Giehl, S. Friedel, N. von Wiren, Plasticity of the Arabidopsis root system under nutrient deficiencies. Plant Physiol 163, 161-179 (2013).

121. A. Medici et al., Identification of Molecular Integrators Shows that Nitrogen Actively Controls the Phosphate Starvation Response in Plants. Plant Cell 31, 1171-1184 (2019).

122. N. Bouain et al., Systems genomics approaches provide new insights into Arabidopsis thaliana root growth regulation under combinatorial mineral nutrient limitation. Plos Genet 15, e1008392 (2019).

123. A. Medici et al., AtNIGT1/HRS1 integrates nitrate and phosphate signals at the Arabidopsis root tip. Nat Commun 6, (2015).

124. Y. Maeda et al., A NIGT1-centred transcriptional cascade regulates nitrate signalling and incorporates phosphorus starvation signals in Arabidopsis. Nat Commun 9, (2018).

125. B. Hu et al., Nitrate-NRT1.1B-SPX4 cascade integrates nitrogen and phosphorus signalling networks in plants (vol 5, pg 401, 2019). Nat Plants 5, 637-637 (2019).

126. S. Li et al., Modulating plant growth-metabolism coordination for sustainable agriculture. Nature 560, 595-+ (2018).

127. R. Gamuyao et al., The protein kinase Pstoll from traditional rice confers tolerance of phosphorus deficiency. Nature 488, 535-539 (2012).

\section{Figure legends}




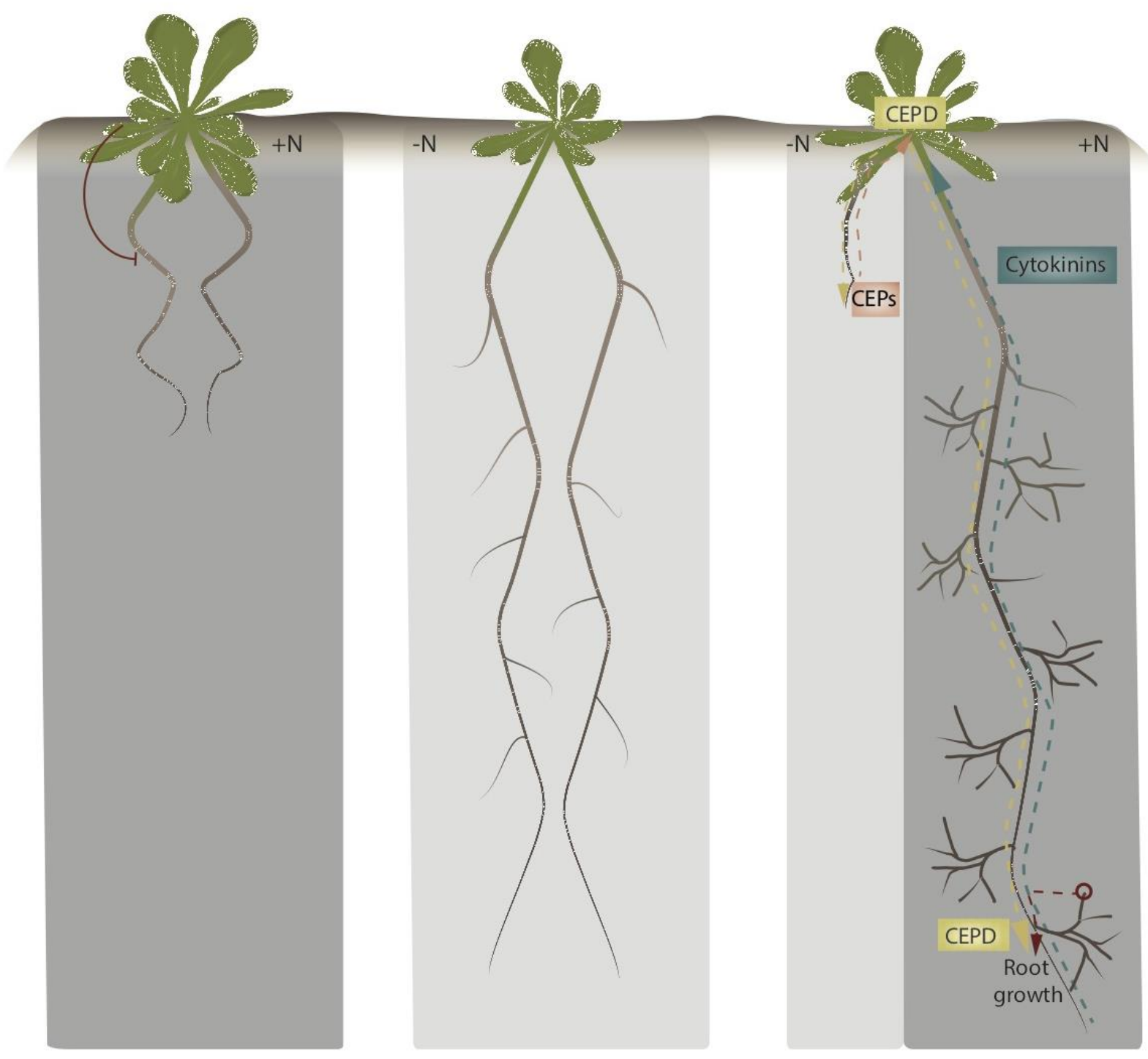

Figure 1: N-response and signaling

(A). A schematic of the root responses of Arabidopsis plants grown in uniform high $\mathrm{N}$ ( $\mathrm{NO}_{3-\text {; }}$; dark grey), uniform low $\mathrm{N}$ (light grey) and differential treatments of high and low $\mathrm{N}$, based on knowledge generated from split-root experiments. Note how the root responses are opposite to the local treatments in uniform versus differential treatments. Underpinning these responses are CEP peptides produced in roots experiencing low $\mathrm{N}$, cytokinins produced in roots experiencing high $\mathrm{N}$, and an N-sufficiency in the shoot, that likely all regulate shoot-root signaling, that at least involves CEPD. Systemic signaling is integrated with local signaling (indicated by red), induced by local perception of $\mathrm{NO}_{3-}$ (see Figure 2). 


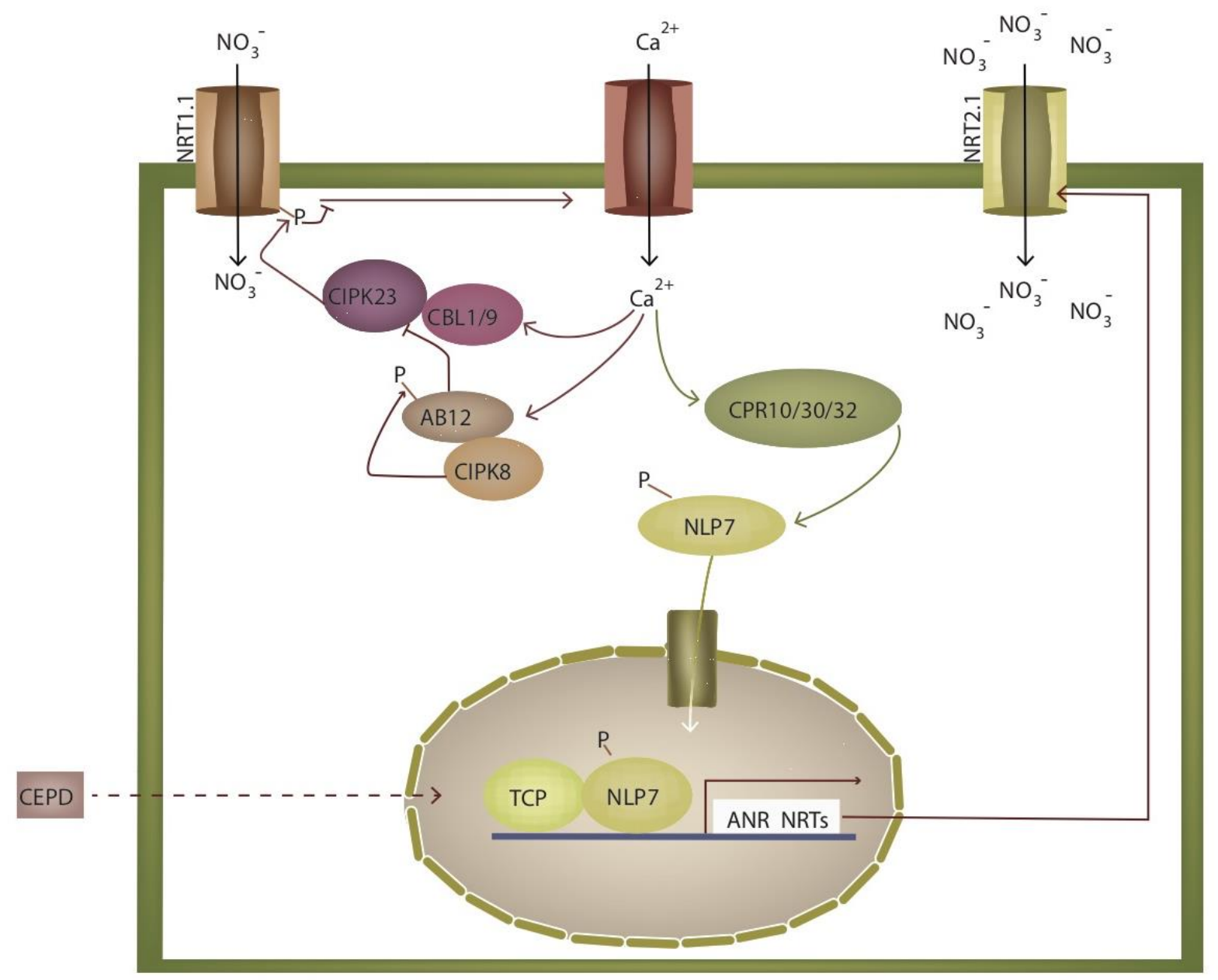

Figure 2: Local NO3- perception and signaling

$\mathrm{NO}_{3}$ - is both perceived and transported by the transceptor NRT1.1. Phosphorylation of NRT1.1 affects both transport and signaling and is controlled by a range of calcium-induced protein kinases (CIPK). NRT1.1 signaling results in a calcium $\left(\mathrm{Ca}_{++}\right)$induced transient across the plasma membrane, that activates a suite of calcium-dependent protein kinases (CPK) that phosphorylate NLP7, allowing its transport into the nucleus. NLP7 interacts with a second transcription factor TCP, that facilitates the integration of the local $\mathrm{NO}_{3-}$ - response with the systemic $\mathrm{N}$-status, likely through the action of CEPD. At least some of the targets of NLP7 are the transcription factor $A N R$, that coordinates root growth according to $\mathrm{N}$-availability, and high affinity $\mathrm{NO}_{3}-$ transporters such as NRT2.2. 


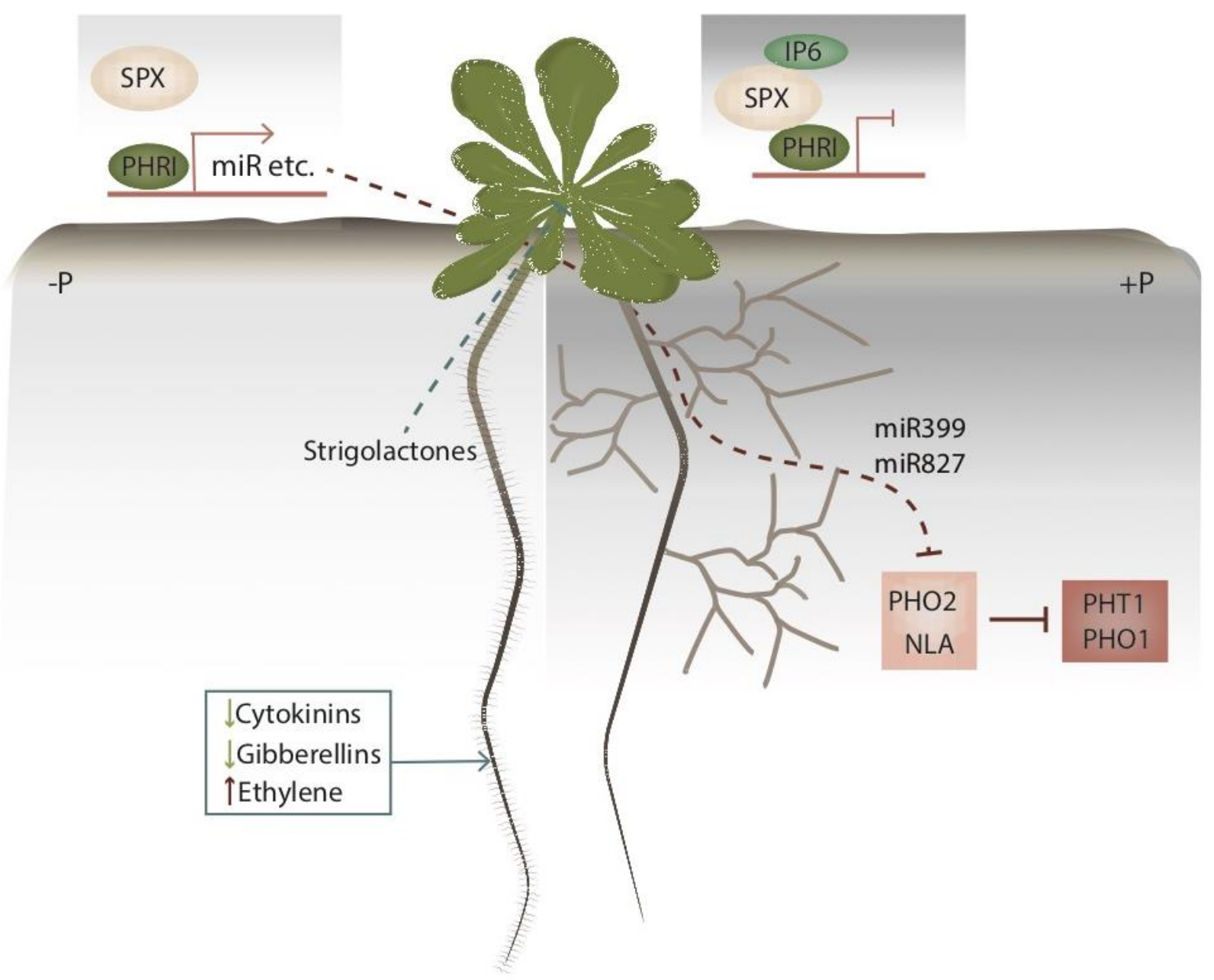

\section{Figure 3: Signaling P-availability}

A summation of signaling processes activated under P-starvation (light grey), versus presence of ample P (dark grey). Multiple hormones are affected by P-availability, as indicated. Auxin levels are also affected in complex ways, not demonstrated in the figure. Systemic signals associated with P-availability are strigolactones, outputs from PHR1 transcriptional regulation and microRNAs, that control the levels of phosphate transporters PHT1 and PHO1. Under Psufficiency PHR1 action is blocked by SPX through its interaction with phytate (IP6) and this suppression is released upon P-starvation. 


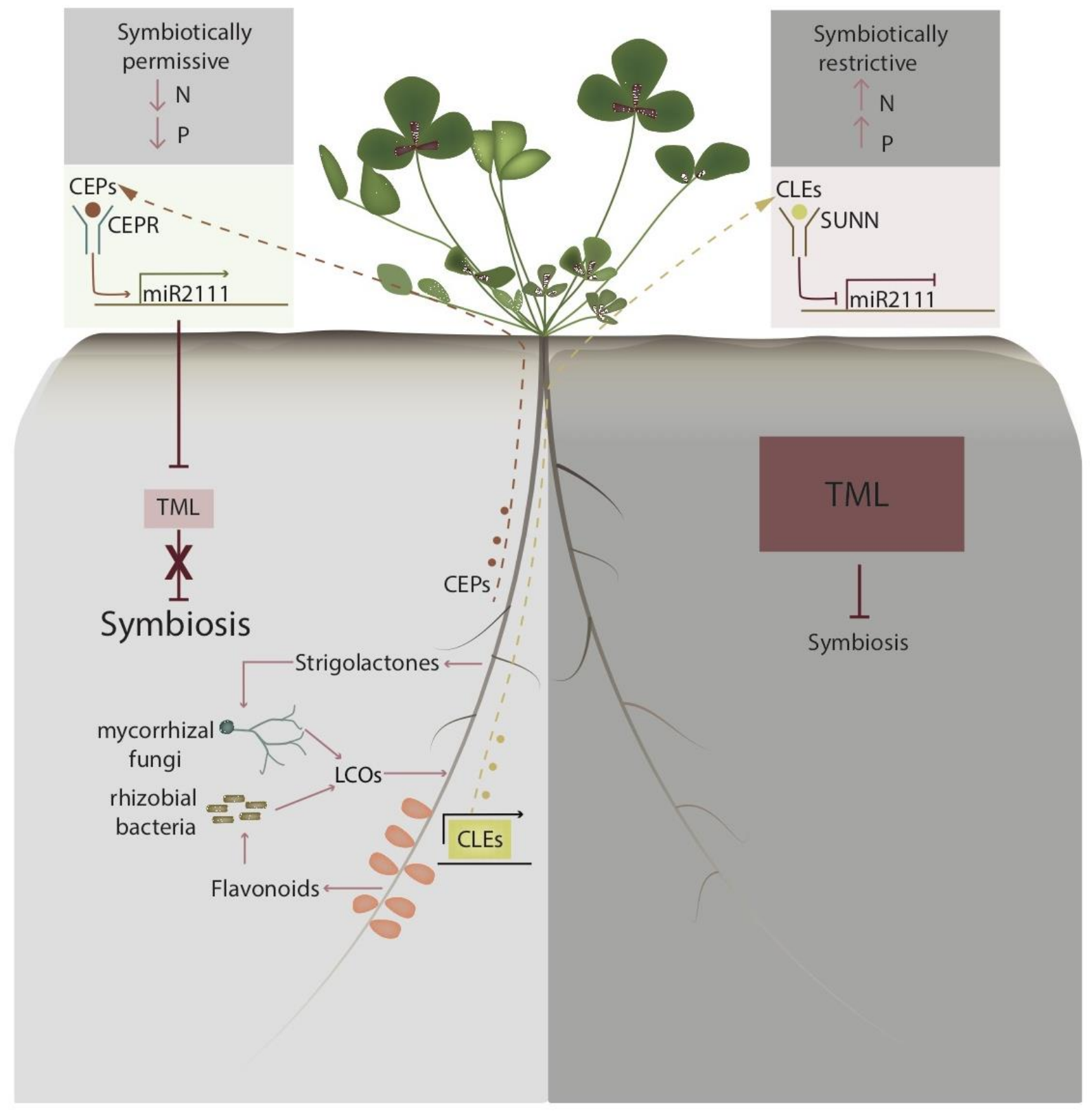

Figure 4: Regulation of symbiotic associations

Plants that enter intracellular symbioses have a symbiotically permissive state under low $\mathrm{N}$ or low $\mathrm{P}$ environments, regulated by CEP peptides, the absence of gibberellins (GA) and the induction of miR2111. The target for miR2111 is Too much love (TML), that is a negative regulator of nodulation. Plants that are permissive to symbiosis perceive lipochitooligosaccharide (LCO) elicitors produced by arbuscular mycorrhizal fungi and $\mathrm{N}$-fixing rhizobial bacteria. Strigolactones, produced under P-starvation, and flavonoids act as plant signals to mycorrhizal fungi or rhizobial bacteria respectively. Symbiotically restrictive conditions are generated either as a result of high nutrients or previous colonization by symbiotic microorganisms leading to the accumulation of CLE peptides, that suppress miR2111 expression via the SUNN/HAR1 receptor. 


\section{One page summary}

\section{Background}

While plants are dependent on the capture of a number of elemental nutrients from the soil, the principle nutrients that limit plant productivity are nitrogen $(\mathrm{N})$ and phosphorus $(\mathrm{P})$. Acquisition of these nutrients is essential for crop performance, but levels of these nutrients in most agricultural soils limit productivity. Thus, these nutrients are typically applied at high concentrations, in the form of inorganic fertilizers, to support global food production. However, their use causes environmental nutrient release, which reduces biodiversity and contributes to climate change and many poor farmers around the world lack the financial resources to recognize the improved crop productivity that fertilizers provide. A more sustainable and equitable agriculture will be less dependent on inorganic fertilizers than the current state of play.

\section{Advances}

Where $\mathrm{N}$ and $\mathrm{P}$ are ample, the ratio of root:shoot biomass allocation can be low with minimal root systems capturing sufficient nutrients. Typically, vegetative growth is extended, allowing resource accumulation and investment in seed production. In environments where these nutrients are limiting overall growth is reduced, but root systems are expanded and colonization by microorganisms is encouraged, to facilitate nutrient capture. Plants can recognize a patchwork of nutrient availability and activate root growth within this patchwork to optimize nutrient capture.

Plant are able to measure multiple facets of nutrient availability: local sensing of nutrients in the soil; roots experiencing nutrient deprivation; roots experiencing high nutrient availability and the total nutrient requirements of the plant. Such sensing involves an integration of root and shoot signaling, with a variety of hormones moving between the root and the shoot to both signal nutrient availability and coordinate plant development. Such root-shoot-root signaling is essential to allow plants to grow into local nutrient patches, but to do so only when there is sufficient need for that nutrient.

A number of microorganisms have unique capabilities for the capture of $\mathrm{N}$ and $\mathrm{P}$ from the environment, for instance $\mathrm{N}$-fixing bacteria can access nitrogen from the atmosphere, something plants are unable to do, while arbuscular mycorrhizal fungi can access insoluble forms of phosphate in the soil, that are mostly inaccessible to plants. Under situations where plants are unable to access $\mathrm{N}$ and $\mathrm{P}$ from their immediate environment, they turn to these microorganisms to allow new sources of these limiting nutrients. It is now apparent that many of the processes that coordinate the plants' developmental response to nutrient availability, also regulate the plants' interaction with microorganisms. These processes regulate the plants receptiveness to its microbial communities, promoting symbiotic associations and restricting immunogenic processes.

\section{Outlook}

Although our understanding of how plants engage with nutrients has advanced, few examples exist where such knowledge has impacted plant performance and this is almost certainly related to the fact that much of our understanding derives from studies in model, not crop, plants. Years of plant breeding under high-nutrient environments have left us with some crop varieties that are poor at optimizing use of limited nutrients. Nonetheless, many processes exist in plants to ensure productivity under poor nutrient conditions, some of which are already accessible in the diversity 
of crop species. There are now many opportunities to utilize the knowledge generated in model systems to optimize the performance of crop plants under nutrient limitation. 